\title{
Isolation and Characterization of Human Gut Bacteria Capable of Extracellular Electron Transport by Electrochemical Techniques
}

\author{
Divya Naradasu ${ }^{1,2 \dagger}$, Waheed Miran ${ }^{1 \dagger}$, Mitsuo Sakamoto ${ }^{3,4}$ and Akihiro Okamoto ${ }^{1,4,5 *}$ \\ ${ }^{1}$ International Center for Materials Nanoarchitectonics (WPI-MANA), National Institute for Materials Science, Tsukuba, Japan, \\ ${ }^{2}$ Department of Advanced Interdisciplinary Studies, Research Center for Advanced Science and Technology, Graduate \\ School of Engineering, The University of Tokyo, Tokyo, Japan, ${ }^{3}$ Microbe Division/Japan Collection of Microorganisms, RIKEN \\ BioResource Research Center, Tsukuba, Japan, ${ }^{4}$ PRIME, Japan Agency for Medical Research and Development (AMED), \\ Tsukuba, Japan, ${ }^{5}$ Center for Sensor and Actuator Material, National Institute for Materials Science (NIMS), Tsukuba, Japan
}

OPEN ACCESS

Edited by:

Fanghua Liu,

Yantai Institute of Coastal Zone Research (CAS), China

Reviewed by:

Suleyman Yildirim,

Istanbul Medipol University, Turkey Wolfgang Buckel,

University of Marburg, Germany

${ }^{*}$ Correspondence:

Akihiro Okamoto OKAMOTO.Akihiro@nims.go.jp

${ }^{\dagger}$ These authors have contributed equally to this work

Specialty section:

This article was submitted to Microbiotechnology, Ecotoxicology and Bioremediation,

a section of the journal

Frontiers in Microbiology

Received: 18 October 2018 Accepted: 17 December 2018

Published: 15 January 2019

Citation

Naradasu D, Miran W Sakamoto M and Okamoto A (2019)

Isolation and Characterization

of Human Gut Bacteria Capable of Extracellular Electron Transport by

Electrochemical Techniques.

Front. Microbiol. 9:3267.

doi: 10.3389/fmicb.2018.03267
Microorganisms are known to exhibit extracellular electron transfer (EET) in a wide variety of habitats. However, as for the human microbiome which significantly impacts our health, the role and importance of EET has not been widely investigated. In this study, we enriched and isolated the EET-capable bacteria from human gut microbes using an electrochemical enrichment method and examined whether the isolates couple EET with anaerobic respiration or fermentation. Upon the use of energy-rich or minimum media (with acetate or lactate) for electrochemical enrichment with the human gut sample at an electrode potential of $+0.4 \mathrm{~V}$ [vs. the standard hydrogen electrode (SHE)], both culture conditions showed significant current production. However, EETcapable pure strains were enriched specifically with minimum media, and subsequent incubation using the $\delta-\mathrm{MnO}_{2}$-agar plate with lactate or acetate led to the isolation of two EET-capable microbial strains, Gut-S1 and Gut-S2, having 99\% of 16S rRNA gene sequence identity with Enterococcus avium (E. avium) and Klebsiella pneumoniae (K. pneumoniae), respectively. While the enrichment involved anaerobic respiration with acetate and lactate, further electrochemistry with $E$. avium and $K$. pneumoniae revealed that the glucose fermentation was also coupled with EET. These results indicate that EET couples not only with anaerobic respiration as found in environmental bacteria, but also with fermentation in the human gut.

Keywords: gut microbes, electromicrobiology, fermentative bacteria, electrochemical enrichment, extracellular electron transfer

\section{INTRODUCTION}

Extracellular electron transfer (EET) mechanisms have evolved in microorganisms as an anaerobic metabolic strategy that can be coupled to the reduction of extracellular solid materials (Myers and Nealson, 1988; Shi et al., 2016). EET mechanisms are proposed to be mediated by cell surface transmembrane cytochromes, exogenous or endogenous soluble redox-active compounds, 
or electrically conductive nanowires (Shi et al., 2016). The significance of EET during anaerobic respiration has provided a reasonable explanation for not only microbial energy conservation and physiology, but also for their interaction with the environment. While EET has been well characterized in terms of its mechanistic basis, mainly in two model bacterial strains, Geobacter sulfurreducens PCA and Shewanella oneidensis MR-1 (Lovley and Phillips, 1988; Myers and Nealson, 1988), electrochemical enrichment studies, combined with $16 \mathrm{~S}$ rRNA-based assessments in a variety of environments, advocate that more physiologically and phylogenetically diverse microorganisms may be capable of using exterior surfaces as electron acceptors. However, it is vital to mention that the survival or enrichment of a microbe on an electrode is not the ultimate evidence for their EET ability, and hence, further electrochemical characterization is essential to probe the EET processes after the isolation of the microbes. A number of enrichments have resulted in the isolation of pure cultures that can accomplish EET with electrodes, indicating that EET may be advantageous in a wide variety of habitats (Zuo et al., 2008; Fedorovich et al., 2009; Logan, 2009; Rowe et al., 2017). Moreover, bacteria that utilize fermentation as their main metabolic pathway have also been isolated and characterized for their EET capabilities (Khan et al., 2012; Zhou et al., 2016; Kumar P. et al., 2018).

In anaerobic environments with substantially reductive condition such as the human gut (Edwards et al., 1985), fermentation is the primary mechanism of microbial metabolism, in which the redox cycling of biological electron carriers, such as nicotinamide adenine dinucleotide (NADH), drives the intracellular oxidation and reduction of organic substrates. As fermentation does not require extracellular electron acceptors for the termination of metabolism, the energy gain under such conditions is potentially lower than that of respiratory metabolism; therefore, the possibility for EET to increase the rate of $\mathrm{NAD}^{+}$regeneration and fermentative metabolism may be important for these microbes to increase their net energy gain and compete with other respiratory bacteria (Okamoto et al., 2017). In fact, a few studies have shown that fermentative gut microbes are capable of EET using soluble electron carrier molecules (Khan et al., 2012; Keogh et al., 2018; Light et al., 2018; Pankratova et al., 2018). However, by simply studying isolated bacterial cultures, it is impossible to examine which bacteria primarily rely on EET-coupled metabolism in the human gut and to study the ecophysiological importance of EET coupled with fermentation, compared to anaerobic respiration, which is also abundant in the gut environment (Rey et al., 2013). Here, we examined the growth competition between fermentative and respiratory bacteria on an electrode surface that enriches for EET-capable bacteria. Specifically, we performed electrochemical enrichment, which was initiated using a diluted gut microbial community, by employing two different medium conditions that biased for either fermentation or anaerobic respiration. The isolated bacterial strains were characterized by electrochemical assays for their metabolism associated with current production and the EET mechanism.

\section{MATERIALS AND METHODS}

\section{Electrochemical Cell Operation and Medium Composition}

Electrochemical measurements were performed in singlechamber and three-electrode reactors. Tin-doped $\mathrm{In}_{2} \mathrm{O}_{3}$ (ITO) grown on a glass substrate by spray pyrolysis deposition was used as the working electrode (WE) having a surface area of $3.1 \mathrm{~cm}^{2}$, and thickness $1.1 \mathrm{~mm}$. The WEs were placed at the bottom of the reactor with sealing gaskets to avoid any leakage. A platinum wire (approximate diameter of $0.1 \mathrm{~mm}$ ) and $\mathrm{Ag} / \mathrm{AgCl}$ (sat. $\mathrm{KCl}$ ) were used as counter and reference electrodes, respectively. Electrochemical experiments were conducted in a COY anaerobic chamber filled with 100\% $\mathrm{N}_{2}$. Electrochemical analysis techniques such as single-potential amperometry (SA) and differential pulse voltammetry (DPV) were measured with an automatic polarization system (VMP3, Bio-Logic Science Instruments). DPV was measured under the following conditions: pulse increment, $5.0 \mathrm{mV}$; pulse amplitude, $50 \mathrm{mV}$; pulse width, $300 \mathrm{~ms}$; and pulse period, $5.0 \mathrm{~s}$. The electrochemical cell was maintained at $37^{\circ} \mathrm{C}$ throughout the experiment and the $\mathrm{WE}$ was poised at $+0.2 \mathrm{~V}$ [vs. $\mathrm{Ag} / \mathrm{AgCl}$ (sat. $\mathrm{KCl})$ ] reference electrode for $\mathrm{SA}$.

Gifu Anaerobic Medium (GAM Broth), which is known for providing reducing conditions and adequate anaerobiosis, was used as an energy rich medium for the enrichment of gut microbes. Defined medium 1 (DM1), used as a minimum medium for EET strains enrichment and initial electrochemical characterization experiments had the following composition $\left(\mathrm{L}^{-1}\right): \mathrm{NH}_{4} \mathrm{Cl}: 1 \mathrm{~g} ; \mathrm{MgCl}_{2} 6 \mathrm{H}_{2} \mathrm{O}: 0.8 \mathrm{~g} ; \mathrm{CaCl}_{2} .2 \mathrm{H}_{2} \mathrm{O}: 0.1 \mathrm{~g}$; $\mathrm{KH}_{2} \mathrm{PO}_{4}: 0.5$ g; yeast extract: $1 \mathrm{~g} ; \mathrm{NaHCO}_{3}: 1 \mathrm{~g}$; trace mineral mix: $10 \mathrm{~mL}$ [with the following composition $\left(\mathrm{L}^{-1}\right)$ : Nitrilotriacetic acid: 1.5 g, $\mathrm{MgSO}_{4} 7 \mathrm{H}_{2} \mathrm{O}: 3 \mathrm{~g}, \mathrm{MnSO}_{4} \mathrm{H}_{2} \mathrm{O}: 0.5 \mathrm{~g}, \mathrm{NaCl}$ : $1 \mathrm{~g}$, FeSO $47 \mathrm{H}_{2} \mathrm{O}: 0.1 \mathrm{~g}, \mathrm{CoSO}_{4} 7 \mathrm{H}_{2} \mathrm{O}: 0.18 \mathrm{~g}, \mathrm{CaCl}_{2} 2 \mathrm{H}_{2} \mathrm{O}$ : $0.1 \mathrm{~g}, \mathrm{ZnSO}_{4} 7 \mathrm{H}_{2} \mathrm{O}: 0.18 \mathrm{~g}, \mathrm{CuSO}_{4} 5 \mathrm{H}_{2} \mathrm{O}: 0.01 \mathrm{~g}, \mathrm{KAl}\left(\mathrm{SO}_{4}\right)_{2}$ $12 \mathrm{H}_{3} \mathrm{BO}_{3}: 0.02 \mathrm{~g}, \mathrm{Na}_{2} \mathrm{MoO}_{4} 2 \mathrm{H}_{2} \mathrm{O}: 0.01 \mathrm{~g}, \mathrm{NiCl}_{2} 6 \mathrm{H}_{2} \mathrm{O}: 0.03 \mathrm{~g}$, $\mathrm{Na}_{2} \mathrm{SeO}^{3} 5 \mathrm{H}_{2} \mathrm{O}: 0.3 \mathrm{mg}, \mathrm{Na}_{2} \mathrm{WO}_{4} 2 \mathrm{H}_{2} \mathrm{O}: 0.4 \mathrm{mg}$; first dissolved the nitrilotriacetic acid and adjusted the $\mathrm{pH}$ to 6.5 with $\mathrm{KOH}$, then added the minerals. Finally, $\mathrm{pH}$ was adjusted to 7.0 with $\mathrm{KOH}$ ] and trace vitamin mix: $10 \mathrm{~mL}$ [with the following composition $\left(\mathrm{L}^{-1}\right)$ : Biotin: $2 \mathrm{mg}$, Folic acid: $2 \mathrm{mg}$, Pyridoxine$\mathrm{HCl}$ : $10 \mathrm{mg}$, Thiamine-HCl: $5 \mathrm{mg}$, Riboflavin: $5 \mathrm{mg}$, Nicotinic acid: $5 \mathrm{mg}$, D-Ca-pantothenate: $5 \mathrm{mg}$, Vitamin B12: $0.1 \mathrm{mg}$, p-Aminobenzoic acid: $5 \mathrm{mg}$, Lipoic acid: $5 \mathrm{mg}$. Acetate $(30 \mathrm{mM})$ or lactate $(30 \mathrm{mM})$ was used as electron donors. DM1 exclusive of $\mathrm{NaHCO}_{3}$, trace mineral, and trace vitamin was autoclaved first, and $\mathrm{pH}$ was adjusted to 7.2 after adding the remaining components. The final medium was filtered using $0.22-\mu \mathrm{m}-$ pore-size filters and deaerated by purging it with $100 \% \mathrm{~N}_{2}$ for $15 \mathrm{~min}$ prior to use for experiments. A fine powder of $\delta-\mathrm{MnO}_{2}$ was synthesized as previously reported (Burdige and Nealson, 1985). Briefly, $\mathrm{Mn}^{2+}$ was oxidized by permanganate under basic conditions, and the product was washed and 
resuspended in distilled water. The solid was freeze-dried for storage, and resuspended in sterilized solution before use in agar plate.

Defined minimum medium 2 (DM2), used as an electrolyte for EET experiments had the following composition $\left(\mathrm{L}^{-1}\right)$ : $\mathrm{NH}_{4} \mathrm{Cl}$ : $1 \mathrm{~g} ; \mathrm{MgCl}_{2} .6 \mathrm{H}_{2} \mathrm{O}: 0.2 \mathrm{~g} ; \mathrm{CaCl}_{2} .2 \mathrm{H}_{2} \mathrm{O}: 0.08$ g; yeast extract: $0.5 \mathrm{~g}$; $\mathrm{NaHCO}_{3}: 2.5 \mathrm{~g}$; NaCl: $10 \mathrm{~g}$; and HEPES buffer: $7.2 \mathrm{~g}$. Acetate $(10 \mathrm{mM})$ or lactate $(10 \mathrm{mM})$ was used as electron donors for EET experiments with DM2. This medium was also autoclaved and deaerated prior to the electrochemical experiments. A total of $5 \mathrm{~mL}$ of medium (including strain culture) was used in the electrochemical reactor for all experiments. During the electrochemical measurements, the reactor was operated at $37^{\circ} \mathrm{C}$ throughout the experiment without any agitation.

\section{Electrochemical Enrichment of Human Gut Sample}

This study was approved by the RIKEN Ethics Committee. A fecal sample was obtained from a healthy volunteer (45-50 years old). A written informed consent agreement signed by the volunteer was obtained before the experiment. A $0.5 \mathrm{~g}$ of fecal sample was suspended in $4.5 \mathrm{~mL}$ of pre-reduced phosphate buffer saline (PBS) and then serially diluted in 10-fold steps. The sample was diluted to a concentration of $10^{-7}(\mathrm{v} / \mathrm{v})$ and $0.1 \mathrm{~mL}$ of this diluted microbial consortium was then added to the electrochemical reactor with $4.9 \mathrm{~mL}$ of GAM medium or DM1 in which the electrolyte temperature was maintained at $37^{\circ} \mathrm{C}$, and the WE was poised at $+0.2 \mathrm{~V}$ throughout the enrichment.

At the end of electrochemical enrichment with GAM, or DM1 having acetate or lactate, the WE surface was washed twice with PBS, and subsequently the electrode-attached biomass from each WE was streaked on separate agar plates which were made with GAM or DM1 having acetate and lactate, and contained 50-60 $\mathrm{mM} \delta-\mathrm{MnO}_{2}$. The plates were incubated at $37^{\circ} \mathrm{C}$ under an $\mathrm{H}_{2} / \mathrm{CO}_{2} / \mathrm{N}_{2}(1: 1: 8 \mathrm{v} / \mathrm{v})$ gas mixture. Given the microbial reduction of $\delta-\mathrm{MnO}_{2}$ generate transparent spot in the dark brown agar plate, this visual clue was used to identify the colony of EET-capable bacteria. The schematics of isolation procedure are shown in Supplementary Figure S1. After 2-4 days of incubation, the colonies that formed the transparent spots were sub-cultured on an Eggerth Gagnon agar (Merck) supplemented with $5 \%$ horse blood at $37^{\circ} \mathrm{C}$ under the same conditions as mentioned above, and single colonies were picked up for further analysis by sequencing. The strains isolated from agar plates having acetate and lactate were named as Gut-S1 and Gut-S2, respectively.

\section{Cell Cultures Harvesting}

The isolated strains Gut-S1 and Gut-S2 were pre-cultivated in $40 \mathrm{~mL}$ of Lysogeny broth (LB) in butyl-rubber-stoppered vials at $37^{\circ} \mathrm{C}$ with an anoxic headspace of $\mathrm{CO}_{2} / \mathrm{N}_{2}(20: 80 \mathrm{v} / \mathrm{v})$. Microbial cultures were harvested in the late exponential phase when the $\mathrm{OD}_{600}$ was about 1.0. The cultures were centrifuged at $7800 \mathrm{rpm}$ at $37^{\circ} \mathrm{C}$ for $10 \mathrm{~min}$ in a $50 \mathrm{~mL}$ falcon tubes. The resultant cell pellet was washed twice with defined media (DM1 or DM2) by resuspending and centrifugation. The resuspended cells in defined media were then added into the reactors to a final $\mathrm{OD}_{600}$ of 0.1 .

\section{Metabolites Determination}

Samples for metabolites were collected from the electrochemical cells at every 8 -h time interval for 24 -h during the electrochemical operations. The collected samples were filtered using $0.22-\mu \mathrm{m}-$ pore-size filters to remove the cells and stored at $-20^{\circ} \mathrm{C}$ until further analysis. For metabolite analysis, samples were diluted 100 times with distilled water. Metabolic products were quantified by using an ion chromatography (IC) system (HIC-20Asuper, Shimadzu Corporation, Japan). Fifty microliter samples were injected, and the anions were analyzed in nonsuppressor mode. Shim-pack IC-A3 and Shim-pack IC-GA3 (Shimadzu Corporation, Japan) were used as the analytical column and guard column, respectively. The mobile phase contained $8 \mathrm{mM}$ p-hydroxybenzoic acid, $3.2 \mathrm{mM}$ Bis-Tris, and $50 \mathrm{mM}$ boric acid, and the flow rate was $1.2 \mathrm{~mL} / \mathrm{min}$. The column temperature was maintained at $40^{\circ} \mathrm{C}$, and the detector (CDD$10 \mathrm{~A}_{\mathrm{SP}}$ ) parameters were set according to the manufacturer's guidelines. Peak area analysis was conducted by Shimadzu analytical workstation software LabSolutions provided by the manufacturer. The standard curves showed sufficiently high linearity $\left(R^{2}=0.999\right)$. Glucose concentrations were measured by using a glucose assay kit (GAGO-20, Sigma-Aldrich) according to the manufacturer's protocol.

\section{DNA Isolation and Phylogenetic Tree}

The discrete colonies that were sub-cultured from the transparent spots of $\delta-\mathrm{MnO}_{2}$ were analyzed by $16 \mathrm{~S}$ rRNA gene sequencing. The forward and reverse primers for PCR were $27 \mathrm{~F}\left(5^{\prime}\right.$-AGA GTT TGA TCC TGG CTC AG-3') and 1492 R (5'-GGT TAC CTT GTT ACG ACT T- $3^{\prime}$ ). The $16 \mathrm{~S}$ rRNA gene sequences were compared with the sequences of closely related strains by using the BLAST program in the GenBank database. These sequence data were deposited in NCBI GenBank under accession numbers MK051424 and MK051423 for Gut-S1 and Gut-S2, respectively. For the construction of a 16S rRNA phylogenetic tree, 16S rRNA sequences were collected from the NCBI nucleotide database, aligned using MUSCLE (Edgar, 2004), and analyzed by the neighbor-joining method (Saitou and Nei, 1987) using Molecular Evolutionary Genetics Analysis package (MEGA, version X.0) (Kumar S. et al., 2018).

\section{Scanning Electron Microscopy}

For the scanning electron microscopy, ITO electrodes were removed from the reactors after performing the electrochemical measurements. Microbial fixation on electrodes was carried out with $2.5 \%$ glutaraldehyde for $10 \mathrm{~min}$ in the dark at room temperature. This was followed by washing three times in $0.1 \mathrm{M}$ phosphate buffer ( $\mathrm{pH} 7.4$ ) for 15 min each. These washed samples were then dehydrated in $30,50,70,90$, and $100 \%$ ethanol gradients (prepared in the $0.1 \mathrm{M}$ buffer) for $15 \mathrm{~min}$ each. Ethanol gradient dehydrated samples were exchanged thrice with 100\% t-butanol and finally freeze-dried under vacuum. The dried samples were coated with platinum and then observed using a Keyence VE-9800 microscope. 


\section{Supernatant Exchange During Current Producing Condition}

The electron transfer mechanism of gut microbes was evaluated using a medium-replacement experiment used to test electron transfer in environmental model microbes $S$. oneidensis MR1 (Marsili et al., 2008). Here, the medium in the electrochemical cell was removed; the biofilm was rinsed with $\mathrm{N}_{2}$-sparged DM twice at each replacement; and the headspace was continuously sparged with $\mathrm{N}_{2}$ during each replacement to avoid the leakage of oxygen into the electrochemical cell. The cell was refilled with $\mathrm{N}_{2}$-sparged sterile medium (10 $\mathrm{mM}$ acetate or lactate). DPV measurements were performed as previously described to detect the redox molecules (Okamoto et al., 2009) before and after supernatant exchanges.

\section{RESULTS}

\section{Electrochemical Enrichment and Isolation of EET-Capable Human Gut Microbes}

For the isolation of fermentative bacteria capable of EET, we initiated electrochemical enrichment in GAM medium with a microbial consortium sample collected from the human gut and diluted it to a concentration of $10^{-7}(\mathrm{v} / \mathrm{v})$ at $37^{\circ} \mathrm{C}$. Current production $\left(I_{\mathrm{c}}\right)$ was measured to be approximately 0.3 $-0.4 \mu \mathrm{A} \mathrm{cm}{ }^{-2}$ for each cycle at around $48 \mathrm{~h}$, and it gradually decreased. After 1 week of incubation, we replaced the spent medium with fresh GAM medium, and after another week of electrochemical incubation, the electrode surface was washed to collect the enriched bacterial cells. Although the subsequent plating resulted in the formation of many colonies, none of the colonies showed transparent spots on the black $\delta-\mathrm{MnO}_{2}$ agar plates, indicating that $\mathrm{MnO}_{2}$ was not reduced, and hence, no colonies of EET-capable bacteria were isolated. This unsuccessful result was probably due to the fact that the GAM medium grew too many fermentative bacterial cells that were incapable of EET in bulk, and the EET-capable bacteria that were potentially enriched on the electrode became a minority.

Next, we used DM1, containing either $30 \mathrm{mM}$ acetate or $30 \mathrm{mM}$ lactate as an electron donor, to enrich for bacteria that can couple EET with anaerobic respiration during the same time frame as the enrichment with the GAM medium. Although the $I_{\mathrm{c}}$ only reached $15-20 \mathrm{nA} \mathrm{cm}-2$ during the DM1 cycles (Supplementary Figures S2a,b), transparent spots were observed on the $\delta-\mathrm{MnO}_{2}$ agar plates, after the incubation of the cells collected from the reactors enriched with either lactate or acetate. All of the colonies with the transparent spots looked identical, and one colony from each plate was analyzed for its 16S rRNA gene sequence. Sequence alignment using NCBI showed that the strain isolated from the acetate-fed reactor belongs to the genus Enterococcus, and the strain isolated from the lactatefed reactor belongs to the genus Klebsiella. Analysis of the $16 \mathrm{~S}$ rRNA gene sequences of the strain Gut-S1 using the GenBank database showed that it has more than $99 \%$ identity with Enterococcus avium, and analysis of the 16S rRNA gene sequences of the lactate-enriched strain Gut-S2 showed that it has more than $99 \%$ identity with Klebsiella pneumoniae. The ribosomal RNA gene sequences of these isolated strains were aligned with representative microbial community sequences from human gut microbes (Enterococcus faecalis and Faecalibacterium prausnitzii) that had been previously reported to have EET capability. The phylogenetic analysis is shown in Figure 1. E. avium and K. pneumoniae, which are similar to Gut-S1 and Gut-S2, are Gram-positive and Gram-negative strains, respectively, and both are fermentative under anaerobic conditions.

\section{Electrochemical Characterization of the Metabolism in the Isolated Strains}

An SA experiment with the isolated strains was performed using the DM1 medium without the addition of acetate or lactate at the start of the incubation (Figure 2). The anodic current was observed to increase once Gut-S1 was added to the reactor with sterile media (Figure 2A), suggesting the microbial capability of EET for the anode in Gut-S1. However, this medium did not contain acetate, and hence, the current production was most likely due to the oxidation of the yeast extract, as no other organic source was present. To our surprise, upon addition of acetate $(30 \mathrm{mM})$, the current production immediately decreased by $10 \%$ and continued to decrease gradually, followed by a short current recovery, suggesting that $30 \mathrm{mM}$ acetate might damage Gut-S1,

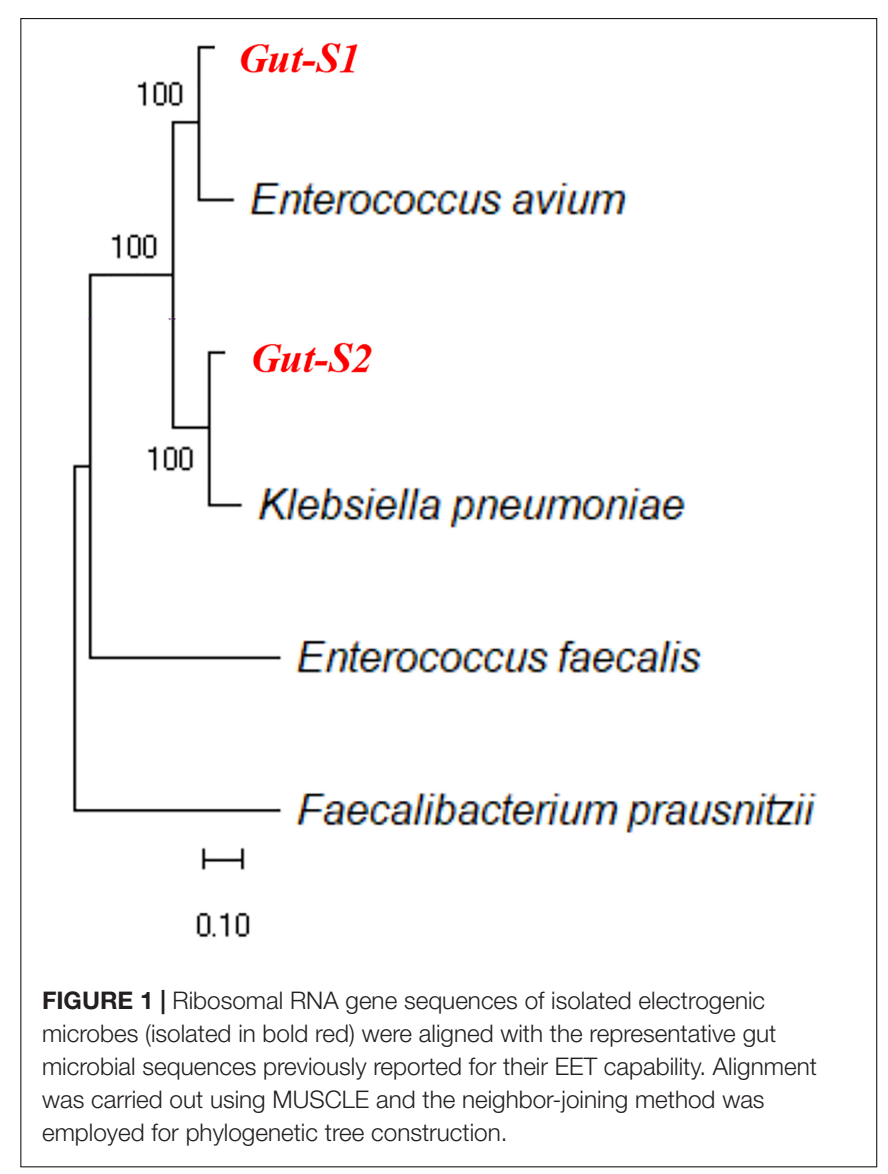




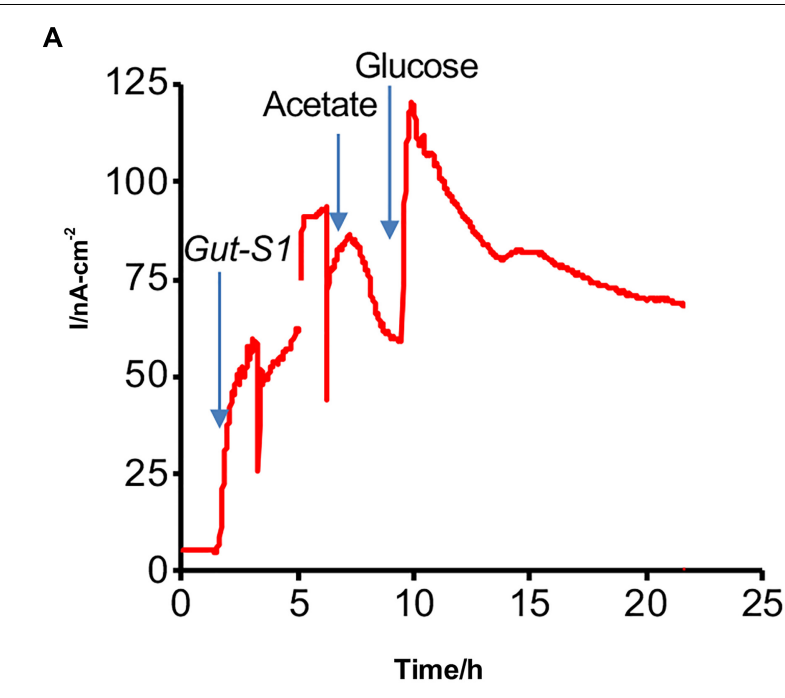

B

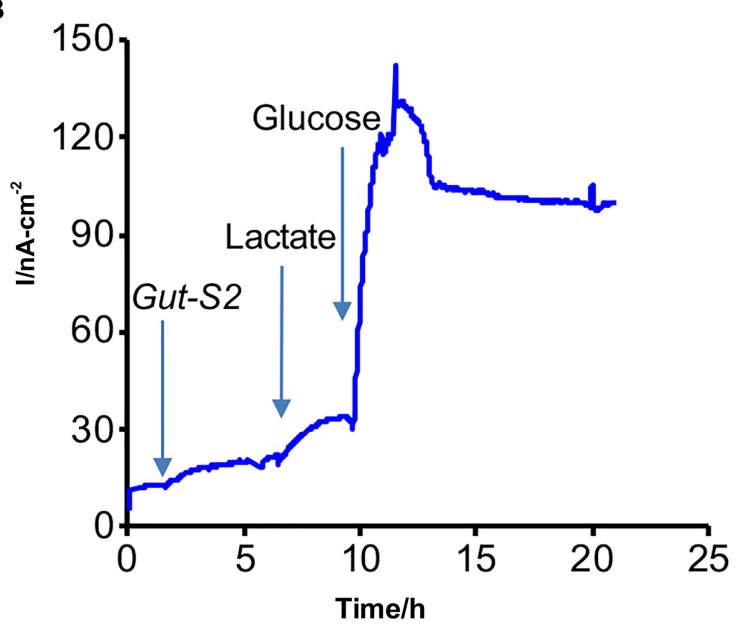

FIGURE 2 | Representative current production versus time in isolated (A) Gut-S1 and (B) Gut-S2 measured with the ITO electrode poised at $0.4 \mathrm{~V}$ vs. $\mathrm{SHE}$, initiated with the sterile DM1 medium containing only yeast extract as a carbon source at time $=0$. At indicated times with arrows, microbes, acetate or lactate, and glucose were added.

although we had been able to use acetate to enrich for the Gut-S1 strain. The current increased to $120 \mathrm{nA} \mathrm{cm}^{-2}$ after the addition of glucose, demonstrating the microbial viability of Gut-S1 and its ability to couple glucose oxidation with current production. These data strongly suggested that Gut-S1 was enriched by the complex organic substrates of yeast extract, rather than by the acetate, implying that there was no microbial strain that could use the abundant acetate to outcompete the bacteria that could utilize the yeast extract.

In contrast, a gradual current increase of $15 \mathrm{nA} \mathrm{cm}-2$ was observed upon lactate addition in the case of Gut-S2 (Figure 2B), suggesting that lactate oxidation contributed to the current production. As glucose addition resulted in an immediate current increase in Gut-S1 (Figure 2A) and Gut-S2 (Figure 2B), the current production may be limited by the rate of the metabolic reaction in both strains. These results indicated
A

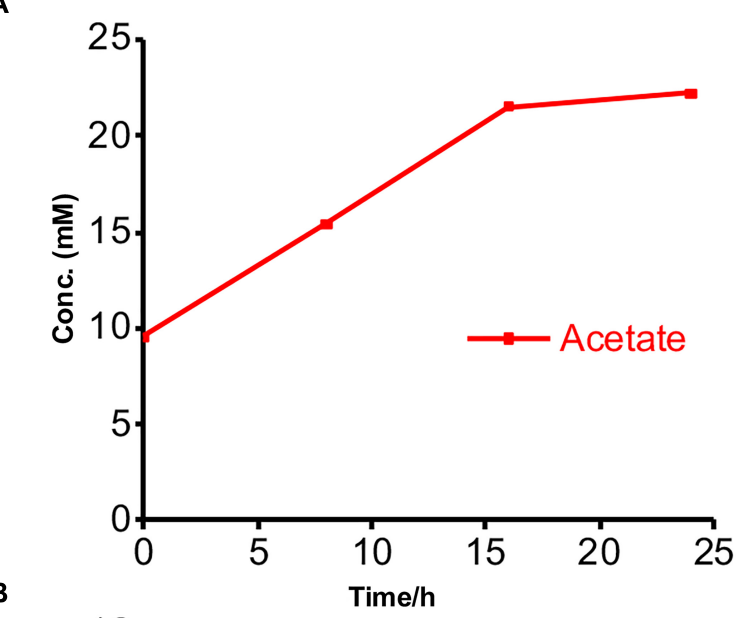

B

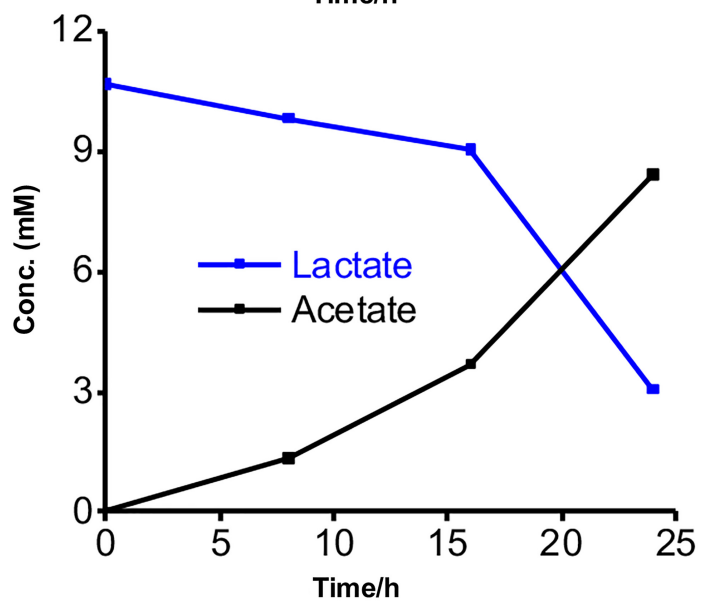

FIGURE 3 | Metabolites concentrations measured at every 8-h time interval during the current production of isolated strains in the presence of acetate or lactate. (A) Acetate production in the presence of Gut-S1 using DM2 containing $10 \mathrm{mM}$ acetate and $0.5 \mathrm{~g} / \mathrm{L}$ yeast extract. (B) Lactate consumption and acetate production in case of Gut-S2 using DM2 containing $10 \mathrm{mM}$ lactate and $0.5 \mathrm{~g} / \mathrm{L}$ yeast extract. Similar tendency was observed in more than two individual experiments.

that the lactate-oxidation metabolism takes the lead role for the enrichment of the Gut-S2 cells on the electrode surface during the electrochemical enrichment.

We further characterized the metabolism of the two strains and the EET mechanisms in these strains by electrochemistry and a metabolite assay in a different minimal medium DM2, which we usually use to characterize the current production capability and EET mechanism of S. oneidensis MR-1 (Okamoto et al., 2013; Saito et al., 2016; Rowe et al., 2017). This medium has a lower yeast extract concentration than DM1 and lacks trace minerals and vitamin solutions. Additionally, the acetate and lactate concentrations were reduced to $10 \mathrm{mM}$ to eliminate the possibility of toxicity to the bacterial cells from the high organic concentrations. No significant change was observed in the current generation with and without acetate in the yeast extractcontaining media for Gut-S1, signifying that EET was not coupled 
A

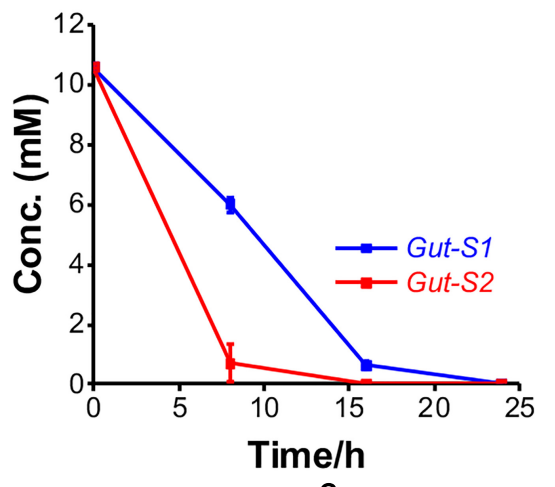

B

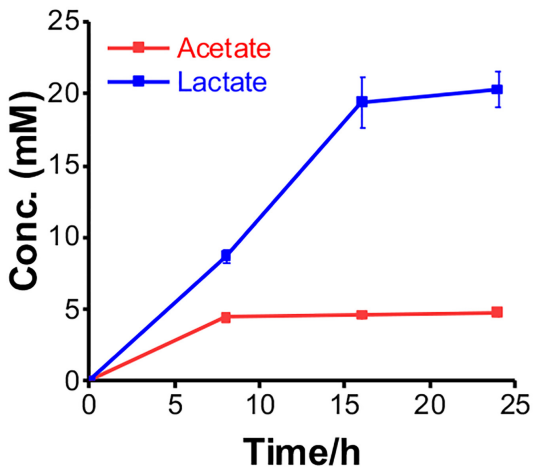

C

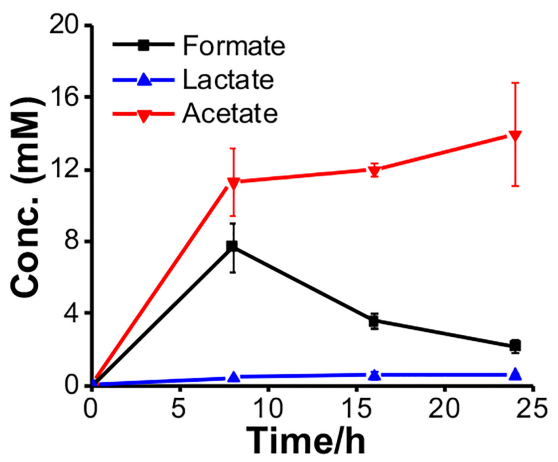

FIGURE 4 | The metabolism of Gut-S1 and Gut-S2 during the current production associated with glucose fermentation. (A) Time course of glucose consumption and its metabolites concentration with (B) Gut-S1 and (C) Gut-S2 during current production using DM2 containing 10 mM glucose. The data shown are the mean values \pm standard deviations of two replicate experiments.

with acetate oxidation in the case of Gut-S1 (Supplementary Figure S3). Furthermore, metabolite quantification for Gut$S 1$ in IC showed that the acetate concentration increased and that it was not consumed, which was most likely due to the production of acetate from the oxidation of the yeast extract (Figure 3A). In the case of Gut-S2, we observed lactate oxidation and acetate production associated with current production (Figure 3B), indicating that lactate was not fermented but anaerobically respired. A slightly lower consumption of lactate, compared to the production of acetate, indicated the oxidation of the yeast extract to produce acetate in Gut-S2. These results demonstrated that Gut-S2 has an EET capability associated with lactate oxidation, which is similar to the anaerobic respiration in the EET model microbial strain, S. oneidensis MR-1.

To examine their ability to couple EET with fermentation, the metabolites were further explored using glucose in the electrochemical system. The consumption and production rates for glucose and the metabolites, respectively, were identified using Gut-S1 and Gut-S2 in DM2. Ten millimolars of glucose was completely consumed by both strains in $24 \mathrm{~h}$, which is 50 $80 \%$ faster than the consumption rate of lactate in S. oneidensis MR-1, indicating considerable microbial activity (Figure 4A). Gut-S1 produced lactate and acetate (Figure 4B), while acetate and formate were the main metabolites for Gut-S2 (Figure 4C). Lactate and formate are known as common end products for bacterial fermentation, and formate can be further oxidized to
$\mathrm{CO}_{2}$ and $\mathrm{H}_{2}$ under anoxic conditions (Lim et al., 2014). The hydrogen produced in electrochemical cells may be oxidized at the ITO electrode surface and may contribute to the current generation by Gut-S2. A very low coulombic efficiency, i.e., less than $0.02 \%$, was observed with both strains, based on the glucose consumption and coulombs generated. Given that this value is much lower than that of environmental bacteria, like S. oneidensis MR-1 (Bretschger et al., 2007), which was also observed in the similar electrochemical set up that was used in our current study (Okamoto et al., 2013), the role of EET in fermentation may be distinct from that of well-studied EET microbes, which has associated EET with anaerobic respiration.

\section{The EET Mechanism in the Two Isolates}

Shewanella oneidensis MR-1 has two potential EET mechanisms: direct electron transfer and indirect electron transport, mediated by a cell-surface enzyme and soluble electron carriers, respectively (Okamoto et al., 2013). To distinguish the two mechanisms for current production, medium exchange experiments have been performed to elucidate the contribution of the soluble electron carriers to the net current production (Marsili et al., 2008). After replacing the spent medium with fresh medium, there was a $20 \%$ decrease in the current for a short period of time. It recovered to the current level before the medium exchange in the case of Gut-S1 (Figure 5A), suggesting a low contribution from the soluble electron carriers 


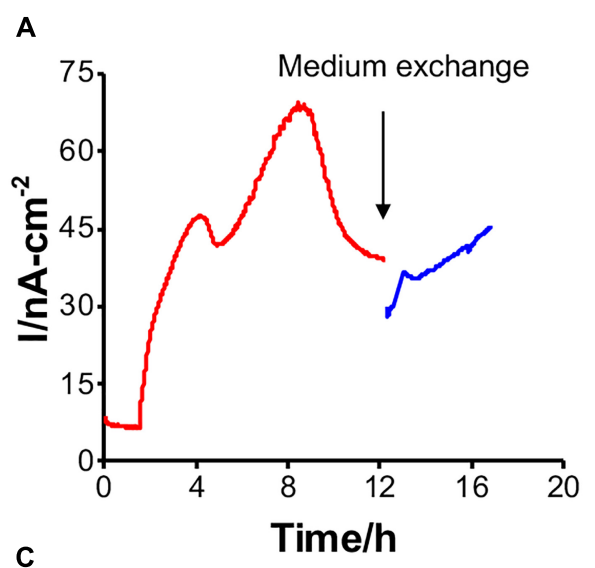

C

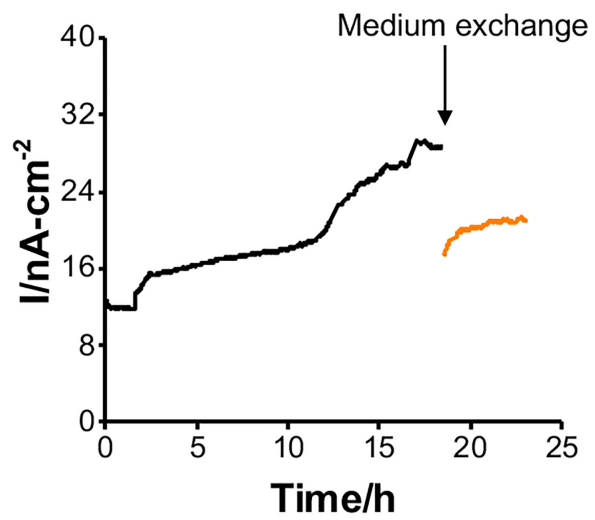

$\mathbf{B}$

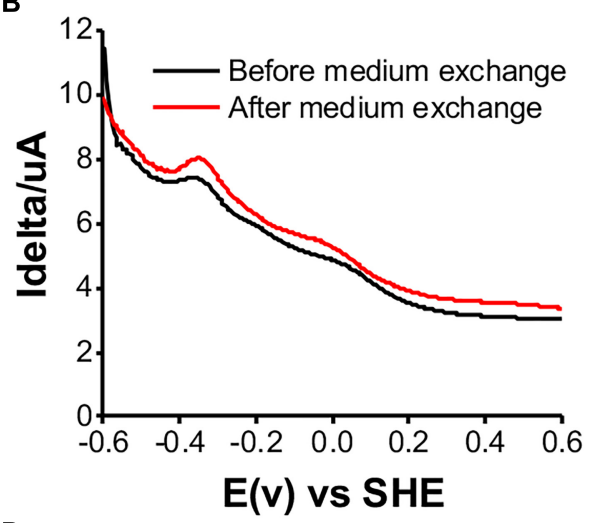

D

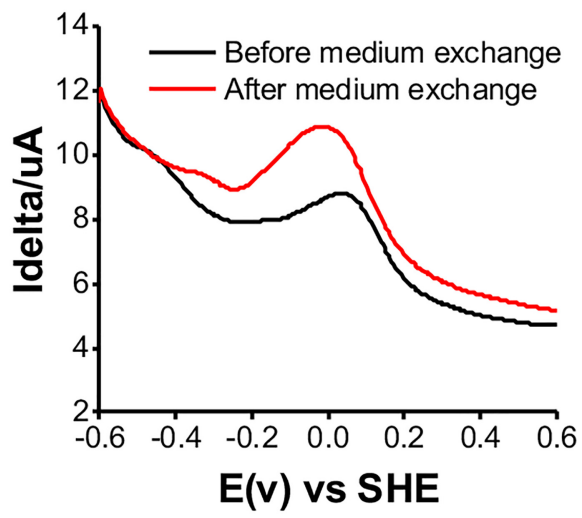

FIGURE 5 | Medium exchange experiments for examining the contribution of soluble electron carrier to the current production, and DP voltammograms before and after medium exchange in reactors with Gut-S1 (A and B) and Gut-S2 (C and $\mathbf{D})$, the similar tendency was observed in more than two experiments.

to the current production. Accordingly, the differential pulse (DP) voltammogram measured before and after the exchange of the electrolyte did not show a significant change, with the oxidative current peaks observed at around -0.35 and $0.0 \mathrm{~V}$ [vs. the standard hydrogen electrode (SHE)] (Figure 5B). In contrast, the current production decreased by approximately $50 \%$ and did not recover to the level from before the medium exchange for Gut-S2 (Figure 5C); the oxidative peak potential also shifted significantly to the positive region in the DP voltammogram (Figure 5D), indicating a larger contribution from the soluble electron shuttle for the current production. Given that the Gut-S2 cells most likely generate hydrogen, we observed the reduction in the formate concentration during current production (Figure 4C), which is a precursor for hydrogen generation. These data suggest that the Gut-S2 current production could be assigned to the oxidation of fermentatively generated hydrogen. However, because a clear peak was still observed at $-0.05 \mathrm{~V}$ (vs. SHE), even after the medium exchange, Gut-S2 may also have a cell surface redox enzyme. Accordingly, for both Gut-S1 and Gut-S2, cellular attachment on the electrode surface was confirmed by SEM analysis after the current production in the presence of glucose (Supplementary Figure S4). Taken together, the different peak positions and intensities of the oxidative peaks in the DP voltammograms suggested that different redox proteins are involved in these two strains.

\section{DISCUSSION}

In this study, the EET ability of isolates from a human gut sample was investigated, and the metabolic pathways were studied. Our data provide evidence that the physiological role of EET coupled with fermentation is somehow distinct from that of anaerobic microbial respiration. The magnitude of the anodic currents reported here, detected from these isolated strains, is significantly lower than those typically reported for the metalreducing microbes usually investigated as EET model systems, and thus, these currents can be easily missed by traditional cultivation strategies. For example, $S$. oneidensis MR-1 produces two orders of magnitude more current than Gut-S1 and Gut-S2 (Xu et al., 2016). The low currents observed here also point to the lower ability of these isolated strains to gain cellular energy from external redox active surfaces. The physiological role of this mode of energy acquisition from external substrates should be further investigated in detail.

The phylogenetic analysis showed that Gut-S1 and Gut-S2 were similar to E. avium and K. pneumoniae, respectively. Both 
strains are facultative anaerobes, but not strict anaerobes, like the majority in the human gut. E. avium is a Gram-positive bacteria and is known as a rare human pathogen, and only a few case series exist (Lee et al., 2004). While the EET capability of this specific strain has not been reported, $K$. pneumoniae has been studied previously for its EET capability. This Gram-negative bacterium was isolated from subterranean forest sediment and investigated using glucose and starch as the carbon sources with successful current generation (Zhang et al., 2008). Furthermore, one study has reported an electron shuttling mechanism in K. pneumoniae based-microbial fuel cells (MFCs) (Deng et al., 2010). In our electrochemical analysis, these strains showed a spontaneous increase in current production with the fermentable carbon source such as glucose, and a less consumption of the nonfermentable carbon sources like acetate/lactate, indicating the fermentation associated EET.

In our study, one of the isolated strains was Gram-negative and the other was Gram-positive, and therefore, they should have different redox proteins for the transfer of the electrons to the electrode surface. Gram-negative bacteria are known to rely on cell surface-exposed cytochromes for the oxidation or reduction of extracellular minerals (Stams and Plugge, 2009; Wegener et al., 2015; White et al., 2016). Much less is known about the electroactivity of gram-positive bacteria than gramnegative ones. The cell envelope of gram-positive bacteria lacks an outer membrane, and the peptidoglycan layer is thicker (20$35 \mathrm{~nm}$ ) (Beeby et al., 2013). Gram-positive bacteria are known to be poor in terms of their current production, but they can donate electrons to an external electrode (Pankratova and Gorton, 2017) and are frequent members of the microbial community in MFCs (Rabaey et al., 2004). A detailed understanding of this aspect may be important for devising strategies for pathogenicity control and the improvement of human health.

\section{REFERENCES}

Beeby, M., Gumbart, J. C., Roux, B., and Jensen, G. J. (2013). Architecture and assembly of the gram-positive cell wall. Mol. Microbiol. 88, 664-672. doi: 10 . $1111 / \mathrm{mmi} .12203$

Bretschger, O., Obraztsova, A., Sturm, C. A., Chang, I. S., Gorby, Y. A., Reed, S. B., et al. (2007). Current production and metal oxide reduction by Shewanella oneidensis MR-1 wild type and mutants. Appl. Environ. Microbiol. 73, 70037012. doi: 10.1128/AEM.01087-07

Burdige, D. J., and Nealson, K. H. (1985). Microbial manganese reduction by enrichment cultures from coastal marine-sediments. Appl. Environ. Microbiol. 50, 491-497.

Deng, L., Li, F., Zhou, S., Huang, D., and Ni, J. (2010). A study of electron-shuttle mechanism in Klebsiella pneumoniae based-microbial fuel cells. Chin. Sci. Bull. 55, 99-104. doi: 10.1007/s11434-009-0563-y

Edgar, R. C. (2004). MUSCLE: multiple sequence alignment with high accuracy and high throughput. Nucleic Acids Res. 32, 1792-1797. doi: 10.1093/nar/gkh340

Edwards, C. A., Duerden, B. I., and Read, N. W. (1985). Metabolism of mixed human colonic bacteria in a continuous culture mimicking the human cecal contents. Gastroenterology 88, 1903-1909. doi: 10.1016/0016-5085(85) 90017-4

Fedorovich, V., Knighton, M. C., Pagaling, E., Ward, F. B., Free, A., and Goryanin, I. (2009). Novel electrochemically active bacterium phylogenetically related to Arcobacter butzleri, isolated from a microbial fuel cell. Appl. Environ. Microbiol. 75, 7326-7334. doi: 10.1128/AEM.01 345-09
In addition to identifying new microbial candidates for EET, this study provides information on the role of EET associated with fermentation, distinct from that of anaerobic respiration. We believe that this new EET mode, coupled with fermentation, may open new windows for biotechnological applications and pathogenicity control models. It should be noted that while the focus here was on gut microbes, similar electrochemically active microorganisms with EET capability may be of interest in the vast range of other human pathogens and the external environment.

\section{AUTHOR CONTRIBUTIONS}

DN, MS, and AO designed and conducted the experiments. WM and $\mathrm{AO}$ wrote the manuscript.

\section{FUNDING}

This study was supported by a grant-in-aid for Research from the Japan Society for Promotion of Science KAKENHI (grant no. 17H04969), the U.S. Office of Naval Research Global (N6290917-1-2038), and PRIME, the Japan Agency for Medical Research and Development (JP18gm6010002 to AO and JP18gm6010007 to MS).

\section{SUPPLEMENTARY MATERIAL}

The Supplementary Material for this article can be found online at: https://www.frontiersin.org/articles/10.3389/fmicb. 2018.03267/full\#supplementary-material

Keogh, D., Lam, L. N., Doyle, L. E., Matysik, A., Pavagadhi, S., Umashankar, S., et al. (2018). Extracellular electron transfer powers Enterococcus faecalis biofilm metabolism. mBio 9:e00626-17. doi: 10.1128/mBio.00626-17

Khan, M. T., Duncan, S. H., Stams, A. J. M., Van Dijl, J. M., Flint, H. J., and Harmsen, H. J. M. (2012). The gut anaerobe Faecalibacterium prausnitzii uses an extracellular electron shuttle to grow at oxic-anoxic interphases. ISME J. 6, 1578-1585. doi: 10.1038/ismej.2012.5

Kumar, P., Chandrasekhar, K., Kumari, A., Sathiyamoorthi, E., and Kim, B. (2018). Electro-fermentation in aid of bioenergy and biopolymers. Energies 11, 343-343. doi: 10.3390/en11020343

Kumar, S., Stecher, G., Li, M., Knyaz, C., and Tamura, K. (2018). MEGA X: molecular evolutionary genetics analysis across computing platforms. Mol. Biol. Evol. 35, 1547-1549. doi: 10.1093/molbev/msy096

Lee, P. P., Ferguson, D. A., and Laffan, J. J. (2004). Vancomycin-resistant Enterococcus avium infections: report of 2 cases and a review of Enterococcus avium infections. Infect. Dis. Clin. Pract. 12, 239-244. doi: 10.1097/01.idc. $0000130887.89740 .7 \mathrm{f}$

Light, S. H., Su, L., Rivera-Lugo, R., Cornejo, J. A., Louie, A., Iavarone, A. T., et al. (2018). A flavin-based extracellular electron transfer mechanism in diverse gram-positive bacteria. Nature 562, 140-144. doi: 10.1038/s41586-018-0498-z

Lim, J. K., Mayer, F., Kang, S. G., and Muller, V. (2014). Energy conservation by oxidation of formate to carbon dioxide and hydrogen via a sodium ion current in a hyperthermophilic archaeon. Proc. Natl. Acad. Sci. U.S.A. 111, 11497-11502. doi: 10.1073/pnas.1407056111

Logan, B. E. (2009). Exoelectrogenic bacteria that power microbial fuel cells. Nat. Rev. Microbiol. 7, 375-381. doi: 10.1038/nrmicro2113 
Lovley, D. R., and Phillips, E. J. P. (1988). Novel mode of microbial energy metabolism: organic carbon oxidation coupled to dissimilatory reduction of iron or manganese. Appl. Environ. Microbiol. 54, 1472-1480.

Marsili, E., Baron, D. B., Shikhare, I. D., Coursolle, D., Gralnick, J. A., and Bond, D. R. (2008). Shewanella secretes flavins that mediate extracellular electron transfer. Proc. Natl. Acad. Sci. U.S.A. 105, 3968-3973. doi: 10.1073/pnas. 0710525105

Myers, C. R., and Nealson, K. H. (1988). Bacterial manganese reduction and growth with manganese oxide as the sole electron acceptor. Science 240, 1319-1321. doi: $10.1126 /$ science. 240.4857 .1319

Okamoto, A., Hashimoto, K., Nealson, K. H., and Nakamura, R. (2013). Rate enhancement of bacterial extracellular electron transport involves bound flavin semiquinones. Proc. Natl. Acad. Sci. U.S.A. 110, 7856-7861. doi: 10.1073/pnas. 1220823110

Okamoto, A., Nakamura, R., Ishii, K., and Hashimoto, K. (2009). In vivo electrochemistry of C-type cytochrome-mediated electron-transfer with chemical marking. Chembiochem 10, 2329-2332. doi: 10.1002/cbic.200900422

Okamoto, A., Tokunou, Y., Kalathil, S., and Hashimoto, K. (2017). Proton transport in the outer-membrane flavocytochrome complex limits the rate of extracellular electron transport. Angew. Chem. Int. Ed. Engl. 56, 9082-9086. doi: 10.1002/anie.201704241

Pankratova, G., and Gorton, L. (2017). Electrochemical communication between living cells and conductive surfaces. Curr. Opin. Electrochem. 5, 193-202. doi: 10.1016/j.coelec.2017.09.013

Pankratova, G., Leech, D., Gorton, L., and Hederstedt, L. (2018). Extracellular electron transfer by the gram-positive bacterium Enterococcus faecalis. Biochemistry 57, 4597-4603. doi: 10.1021/acs.biochem.8b00600

Rabaey, K., Boon, N., Siciliano, S. D., Verhaege, M., and Verstraete, W. (2004). Biofuel cells select for microbial consortia that self-mediate electron transfer. Appl. Environ. Microbiol. 70, 5373-5382. doi: 10.1128/AEM.70.9.5373-5382. 2004

Rey, F. E., Gonzalez, M. D., Cheng, J. Y., Wu, M., Ahern, P. P., and Gordon, J. I. (2013). Metabolic niche of a prominent sulfate-reducing human gut bacterium. Proc. Natl. Acad. Sci. U.S.A. 110, 13582-13587. doi: 10.1073/pnas.131252 4110

Rowe, A. R., Yoshimura, M., LaRowe, D. E., Bird, L. J., Amend, J. P., Hashimoto, K., et al. (2017). In situ electrochemical enrichment and isolation of a magnetitereducing bacterium from a high $\mathrm{pH}$ serpentinizing spring. Environ. Microbiol. 19, 2272-2285. doi: 10.1111/1462-2920.13723

Saito, J., Hashimoto, K., and Okamoto, A. (2016). Flavin as an indicator of the ratelimiting factor for microbial current production in Shewanella oneidensis MR-1. Electrochim. Acta 216, 261-265. doi: 10.1016/j.electacta.2016.09.002
Saitou, N., and Nei, M. (1987). The neighbor-joining method: a new method for reconstructing phylogenetic trees. Mol. Biol. Evol. 4, 406-425.

Shi, L., Dong, H., Reguera, G., Beyenal, H., Lu, A., Liu, J., et al. (2016). Extracellular electron transfer mechanisms between microorganisms and minerals. Nat. Rev. Microbiol. 14, 651-662. doi: 10.1038/nrmicro.2016.93

Stams, A. J. M., and Plugge, C. M. (2009). Electron transfer in syntrophic communities of anaerobic bacteria and archaea. Nat. Rev. Microbiol. 7, 568-577. doi: $10.1038 /$ nrmicro2166

Wegener, G., Krukenberg, V., Riedel, D., Tegetmeyer, H. E., and Boetius, A. (2015). Intercellular wiring enables electron transfer between methanotrophic archaea and bacteria. Nature 526, 587-590. doi: 10.1038/nature15733

White, G. F., Edwards, M. J., Gomez-Perez, L., Richardson, D. J., Butt, J. N., and Clarke, T. A. (2016). Mechanisms of bacterial extracellular electron exchange. Adv. Microb. Physiol. 68, 87-138. doi: 10.1016/bs.ampbs.2016. 02.002

Xu, S., Jangir, Y., and El-Naggar, M. Y. (2016). Disentangling the roles of free and cytochrome-bound flavins in extracellular electron transport from Shewanella oneidensis MR-1. Electrochim. Acta 198, 49-55. doi: 10.1016/j.electacta.2016. 03.074

Zhang, L. X., Zhou, S. G., Zhuang, L., Li, W. S., Zhang, J. T., Lu, N., et al. (2008). Microbial fuel cell based on Klebsiella pneumoniae biofilm. Electrochem. Commun. 10, 1641-1643. doi: 10.1016/j.elecom.2008.08.030

Zhou, L., Deng, D., Zhang, D., Chen, Q., Kang, J., Fan, N., et al. (2016). Microbial electricity generation and isolation of exoelectrogenic bacteria based on petroleum hydrocarbon-contaminated soil. Electroanalysis 28, 1510-1516. doi: 10.1002/elan.201501052

Zuo, Y., Xing, D., Regan, J. M., and Logan, B. E. (2008). Isolation of the exoelectrogenic bacterium Ochrobactrum anthropi YZ-1 by using a U-tube microbial fuel cell. Appl. Environ. Microbiol. 74, 3130-3137. doi: 10.1128/AEM. 02732-07

Conflict of Interest Statement: The authors declare that the research was conducted in the absence of any commercial or financial relationships that could be construed as a potential conflict of interest.

Copyright (C) 2019 Naradasu, Miran, Sakamoto and Okamoto. This is an open-access article distributed under the terms of the Creative Commons Attribution License (CC BY). The use, distribution or reproduction in other forums is permitted, provided the original author(s) and the copyright owner(s) are credited and that the original publication in this journal is cited, in accordance with accepted academic practice. No use, distribution or reproduction is permitted which does not comply with these terms. 